\title{
Surveillance of Cardio Vascular Risk Factors Among Patients Undergoing Coronary Artery Bypass Surgery
}

\author{
Shumaila Furnaz* and Hasanat Sharif \\ Surgery, Aga khan university and hospital, Karachi, Pakistan
}

\section{Objective}

To investigate the prevalence of cardiovascular risk factors among patients undergoing elective Coronary Artery Bypass Graft surgery $(\mathrm{CABG})$ in Karachi, Pakistan.

\section{Introduction}

According to world health organization report 2011, coronary artery diseases are the number one cause of death globally: more people die annually from coronary artery diseases than from any other cause.

An estimated 17.3 million people died from coronary artery diseases in 2008, representing $30 \%$ of all global deaths. Of these deaths, an estimated 7.3 million were due to coronary heart disease and 6.2 million were due to stroke. Low- and middle-income countries are disproportionally affected: over $80 \%$ of coronary artery diseases deaths take place in low- and middle-income countries and occur almost equally in men and women.

Populations living in low and middle income countries are exposed to more risk factors associated with coronary artery disease as well as other non-communicable diseases and are less exposed to prevention efforts than people in high income countries.

\section{Methods}

Cardiothoracic surgery quality improvement is a core value of healthcare provision. In order to improve quality of care, information on key indicators needs to be systematically collected and maintained.

In 2006, the cardiothoracic department at Aga Khan University developed an infrastructure that would enable us to answer the more challenging research queries in cardiac surgery practice.

The resulting electronic cardiothoracic database is based on the European Association of Cardiothoracic Surgeons database and the Society of Thoracic Surgeons database.

We chose the following aspects of patient care to be included in the database form: pre-surgery patient condition and medications, anesthesia information, perfusion information, surgery information, recovery information, status of the patient atdischarge,30-days and 365-days post-surgery follow-up information .

Information was collected through structured questionnaire by trained data abstractor and entered into Microsoft Access software and analyzed in SPSS(Statistical package of social sciences) software.

\section{Results}

In this prospective study 2073 undergoing elective CABG were included. Mean age of the patients was $54.85 \pm 9.7$ years, $14.7 \%$ of patients were females. Prevalence of risk factors among the study population, included: $47.10 \%$ were overweight, $14.7 \%$ were obese, $47.7 \%$ were diabetic, $69.50 \%$ were hypertensive, $50.20 \%$ were dyslipidemia, $47.7 \%$ were as smokers, $9.2 \%$ were in renal failure, addition, 53\% of patients had family history of coronary artery disease, $46.7 \%$ had a history of myocardial Infarction (MI). The,operative mortality rate was $0.3 \%$.,Post surgery complications included renal failure in $14.8 \%$ of patients, arrhythmias in $7 \%$, reoperation in $2.3 \%$, prolonged ventilation in $3.1 \%$.

\section{Conclusions}

There is a high prevalence of risk factors like dyslipidemia, hypertension, diabetes and smoking for ischemic heart disease in our population. Once we establish this fact we will work to control the risk factors and reduce the burden of disease so that's why this study is being done

\section{Keywords}

Coronary artery bypass grafting; Risk factors; surveillance

\section{Acknowledgments}

Mubashir Khan, Hasanat Sharif

*Shumaila Furnaz

E-mail: shumaila.furnaz@aku.edu 